\title{
Fabrication of Glucose Sensitive Actuator for DDS Micro valve
}

\author{
N. A. DZULKEFLI ${ }^{1, a}, B \cdot B A I S^{2, b}$ and B. Y. MAJLIS ${ }^{3, c}$ \\ ${ }^{1,2,3}$ Institute of Microengineering and Nanoelectronics (IMEN), Universiti Kebangsaan Malaysia \\ 43600, Bangi, Selangor, Malaysia \\ anurazrina_rin@yahoo.co.uk'badariah@vlsi.eng.ukm.my' cburhan@vlsi.eng.ukm.my
}

Keywords: Micro valve, micro channel, glucose actuator, MEMS

\begin{abstract}
A glucose sensitive actuator for drug delivery system (DDS) consists of silicon boss, micro channel and outlet of the microvalve. The microchannel is essential to carry liquid samples in the system. In this paper, two types of microchannel, rectangular and trapezoidal, were fabricated using anisotropic etching of Deep-RIE and wet chemical etching using $\mathrm{KOH}$ solution, respectively. Fabrication were done for micro channels with cross sectional width of $100-120 \mu \mathrm{m}$ width and length of $2 \mathrm{~mm}$.
\end{abstract}

\section{Introduction/Theory}

Glucose-Microvalve is a BioMEMS application in drug delivery system which allows better control of drug release through human bodies. Diabetic patients usually need long term diet management along with daily insulin injection to control their glucose concentration. With this microvalve, the insulin can be released cordially to human body automatically based on the glucose concentration. This hydrogel-actuated microvalve is responds to the changes in the concentration of glucose in an external liquid environment. The hydrogel which has high water permeability will react with the glucose and swell due to the acid-base equilibrium. The used of responsive hydrogel materials which to regulate flow, eliminates the need for both external power and control and complex fabrication schemes. The thinner the hydrogel, the smaller deflection of the membrane will produce. Decreasing the thickness of the hydrogel should be compatible with the force needed to open the microvalve. The research on micro valve for micro fluidic actuator applications has been increasing steadily during the last years. This has resulted in a number of publications regarding these glucose actuators for micro valve [1-3].

Thus the design and fabrication technique of this glucose actuator were developed to meet the hydrogel requirement; so that this actuator microvalve will be successfully operated accordingly. The silicon boss were forced by slicon membrane by the hydrogel reaction,were design to be smaller than membrane. Microchannels are essential to realizing the necessary functionalities of a microfluidic system, as they connect different elements, deliver the fluids and accomodate the reaction . According to standard theory, the stationary laminar and fully developed flow, $\varphi$ through a channel with a constant cross section is described by Schadel [4]:

$$
\varphi=\frac{1}{C_{R}} \frac{A}{\eta L}(\Delta \rho)
$$

where $\Delta \rho$ denotes the pressure drop at the channel, A the channel cross section, $L$ the channel length, $\eta$ is the viscosity of the fluidic and $C R$ is introduced as a geometry coefficient, which summarizes the influence of the shape of the cross section. It can be derived for the Poiseuilles number, Po

$$
C_{R}=\frac{U^{2}}{8 A} P_{0}
$$

where $\mathrm{A}$ is the cross section and $\mathrm{U}$ the wetted perimeter. 


\section{Design and Fabrication Process}

A glucose sensitive actuator for drug delivery system consists of silicon boss, microchannel and outlet of the microvalve as shown in Fig. 1. The silicon boss, which has direct contact to the membrane will deflect due to the swelling reaction of the glucose hydrogel. Silicon rubber with a Young modulus of $1.18 \mathrm{MPa}$ was used to simulate the diaphragm. It's high tear resistance $(42 \mathrm{kN} / \mathrm{m})$ and capability to sustain elongation as high as $1000 \%$, allowed very large deflection and results in the insulin flowing through the microchannel from outlet to inlet [5].

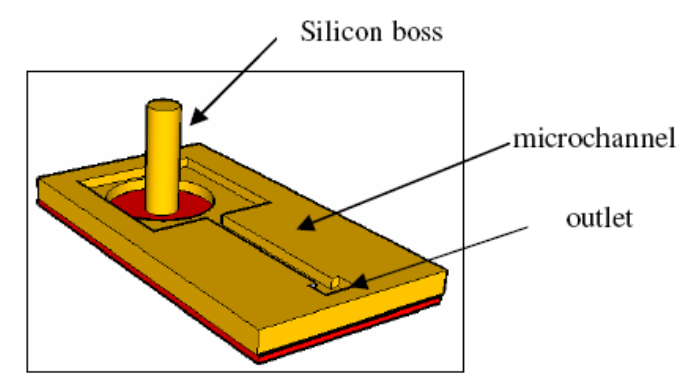

Fig. 1 Design of glucose sensitive actuator part for DDS microvalve

a)

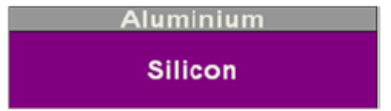

b)

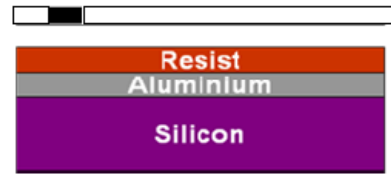

c)

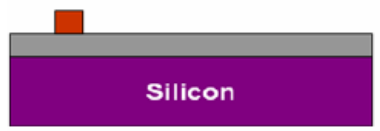

d)

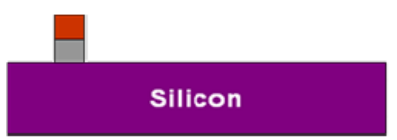

e)

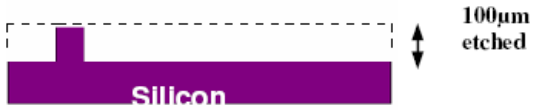

f)

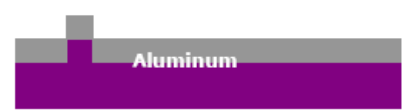

g)

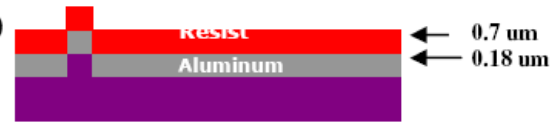

h)

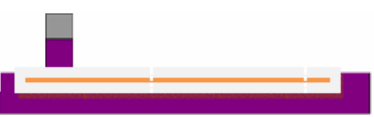

Fig. 2: The fabrication process for the rectangular microchannel

Fig. 2 shows the fabrication process for the rectangular microchannel. It starts with a $<100>$ silicon wafer evaporated with Aluminum using the metal evaporator (Fig. 2a). Then a layer of photo resist is spin coated onto the Aluminium and patterned (Fig. $2 b$ \& 2c). Subsequently, the patterned Aluminum was removed by using $\mathrm{H} 3 \mathrm{PO} 4 / \mathrm{HNO} 3 / \mathrm{H} 2 \mathrm{O}$ etchant and the remining Aluminium will be used as the mask for Deep-RIE etching in order to fabricate the silicon boss (Fig. 2d). Then, Deep-RIE etching were performed on the silicon and the Aluminium and the resist were removed (Fig. 2e). The silicon boss is then re-evaporated with Aluminum and re-patterned with a resist to create the micro channel and outlet mask (Fig. $2 \mathrm{f} \& 2 \mathrm{~g}$ ). Finally, the wafer was etched using Deep-RIE to obtain the rectangular micro channel and outlet (Fig. 2h). The estimated etch rate is $2.5 \mu \mathrm{m} / \mathrm{min}$. 


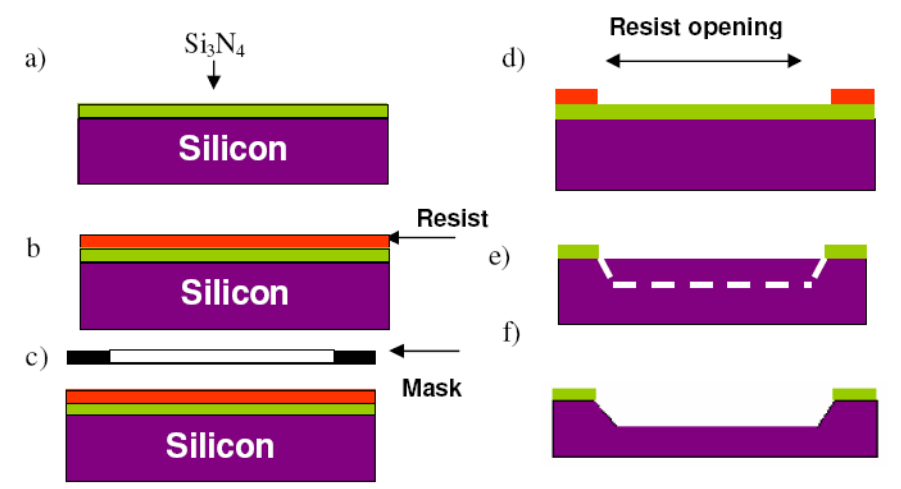

Fig. 3: Fabrication process for the trapezoidal microchannel

Fig. 3 shows the fabrication process for the trapezoidal microchannel. For this microchannel, a $<100>$ silicon wafer was coated with $200 \mathrm{~nm}$ of Si3N4 (Fig 3a). It begins with patterning the wafer with the micro channel and outlet mask (Fig 3b\&3c). The unexposed resist and Si3N4 layer was removed using the Deep-RIE to form the opening on the silicon wafer for $\mathrm{KOH}$ etching (Fig 3d). The etch rate for Si3N4 is $400 \mathrm{~nm} / \mathrm{sec}$. A $35 \% \mathrm{KOH}$ solution at $80{ }^{\circ} \mathrm{C}$ was chosen as etching parameter which enable controllable etch process with an etch rate of about $1 \mu \mathrm{m} / \mathrm{min}$ (Fig 3e\&3f).

\section{Results and Discussion}

Fig. 4 shows the cross section of rectangular micro channel with the outlet fabricated using the Deep-RIE process. From Fig. 4, it was found that the etch surface inside the microchannel was smooth with $90^{\circ}$ slope in the wall of the microchannel. However, it can be seen that the etch surface at the outlet area was not as smooth. This surface roughness can be eliminated by reducing the oxygen composition in the etching recipe.

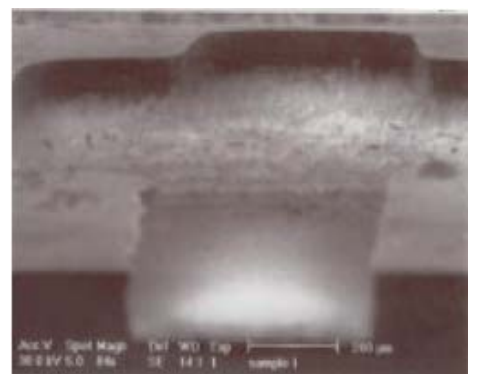

Fig. 4 Cross section of rectangular microchannel

Fig. 5 shows the top view of the trapezoidal microchannel and outlet fabricated with $\mathrm{KOH}$ etching process. Three micro channels with various widths were fabricated; namely $100 \mu \mathrm{m}, 150 \mu \mathrm{m}$ and 200 $\mu \mathrm{m}$. The length of the micro channel was fixed at $2 \mathrm{~mm}$. From Fig. 5 it can be seen that the wider the width of the microchannel, the smoother the etching surface. This is because the etching process attacked the corner of Si3N4 which produce rougher surface and sides.

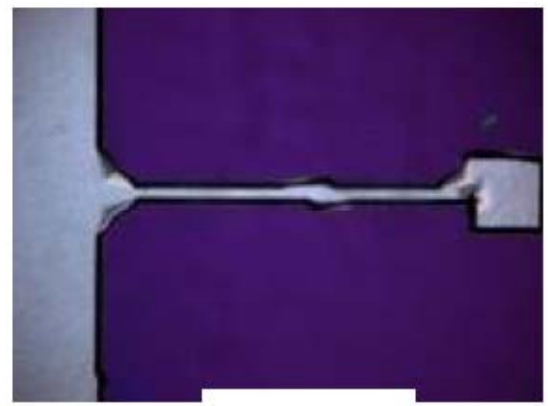

(a)

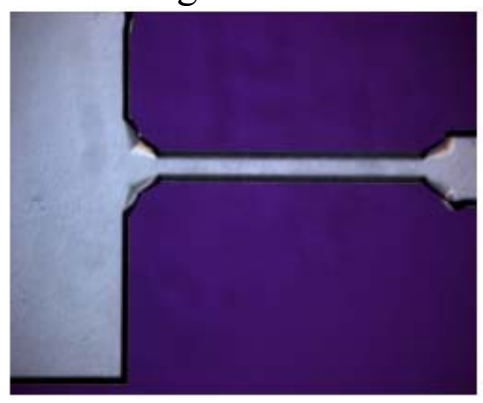

(b)

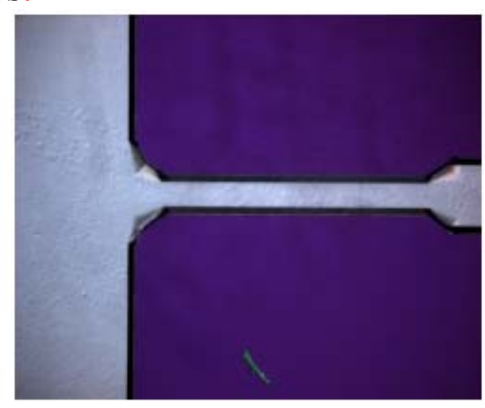

(c)

Fig. 5 Trapezoidal micro channel with width of (a) $100 \mu \mathrm{m}$ (b) $150 \mu \mathrm{m}$ and (c) $200 \mu \mathrm{m}$ 
Fig 6a shows the SEM images of straight silicon boss obtained from the Deep-RIE process. To perform this design, the recipe used to run the Deep-RIE must be accurate and stable and the etch rate need to be calculated to estimate the ultimate time for running the etching process. Figure $6 \mathrm{~b}$ shows the defect of the silicon boss obtained from the unstable Deep-RIE process. End of the boss have slope which suppose to be $90^{\circ} \theta$ straight. At close view (figure $6 \mathrm{c}$ ), it can be seen that at the slope, the Aluminum Deep-RIE mask, was covering end of the boss. This waste might be the reason for this defect, where it prevents the plasma to etch directly to the silicon surface.

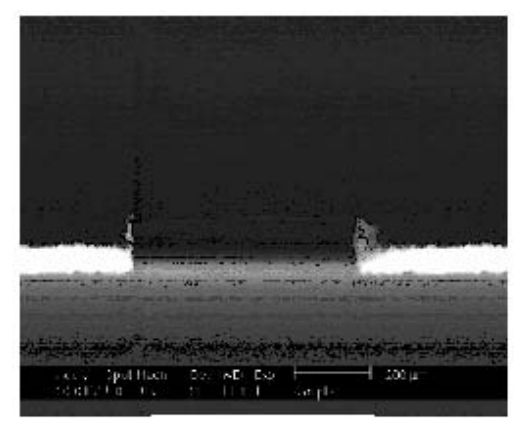

(a)

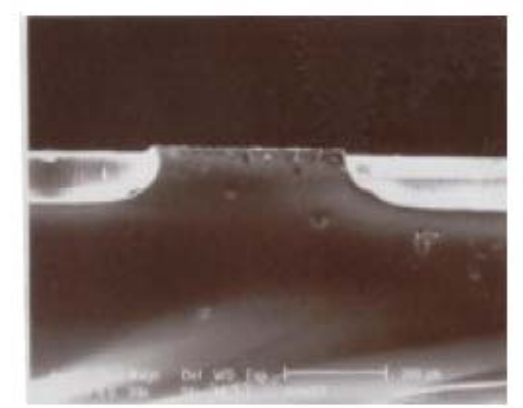

(b)

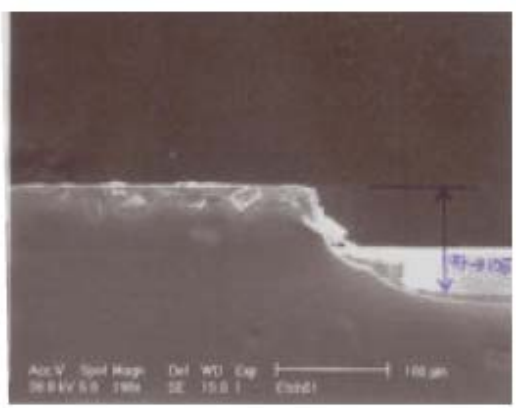

(c)

Fig. 6 Silicon boss (a) perfect boss (b) defect boss and (c) cross view of defect boss

\section{Conclusion}

The fabrication of glucose sensitive actuator for DDS micro valve was studied. Two types of micro channels were fabricated using anisotropic etching of Deep-RIE and wet chemical etching using $\mathrm{KOH}$ solution. It was found that both rectangular and trapezoidal micro channel can be fabricated. However, the process steps need to be optimized in order to obtain a smoother micro channel. In future work, the fabrication process steps and recipe will be optimized.

\section{References}

[1] R. H. Liu, Q. Yu and D. J. Beebe,VOL. 11, NO. 1, pp. 45-53, 2002.

[2] A.Baldi, Y. Gu, P. E. Loftness, R. A. Siegel and B. Ziaie. 12, NO. 5, pp. 613-621, Oct. 2003

[3] Q.Yu, J.M Bauer, J.S. Moore and D.J Beebe, "International IEEE-Microtechnology,France, 2000

[4] Richter, P.Woias, D.Weib: Journal Sensor and Actuators A Vol 62. (1997), p. 480-483

[5] Masoomeh Tehranirokh,Burhanuddin Yeop Majlis,Badariah Bais, "Design of micromechanical system utilizing hydrogel force for controlling a microvalve“.Proc ICSE Int. Semiconductor 2008. 